\title{
AlN/Pt/LN-Y128 packageless acoustic wave temperature sensor
}

\author{
Cécile Floer, Member, IEEE, Omar Elmazria, Senior Member, IEEE, Natalya Naumenko, Florian \\ Bartoli, Jaafar Ghanbaja, Thierry Aubert and Sami Hage-Ali, Member, IEEE
}

\begin{abstract}
Batteryless, wireless and packageless acoustic wave sensors are particularly desirable for harsh high temperature environments. In this paper, an acoustic wave sensor based on a lithium niobate $\left(Y+128^{\circ}\right.$ cut, abbreviated $\left.\mathrm{LN}-\mathrm{Y} 128\right)$ substrate with a buried platinum interdigital transducer (IDT) in an aluminum nitride (AIN) overlayer is investigated. Previously, it was demonstrated theoretically that due to the specific properties of LN-Y128, Rayleigh-type guided waves can propagate at the AIN/IDT(Pt)/LN-Y128 interface. Here, this structure is, for the first time, studied experimentally, including the growth and properties of the AIN layer onto irregular platinum IDTs. Both Shear Horizontal and Rayleigh type waves have been identified after the AIN deposition and the velocities are consistent with the fitted SDA-FEM-SDA simulations. Electrical measurements with a surface perturbation and temperature measurements show that the AIN/IDT(Pt)/LN-Y128 bilayer structure is promising as a packageless high temperature sensor.
\end{abstract}

Index Terms - Packageless, guided acoustic wave, temperature

\section{INTRODUCTION}

$\mathrm{S}$ urface acoustic wave (SAW) devices are widely used as filters, resonators or delay lines in electronic systems for a wide range of applications such as mobile communications (cell phones), TVs, radars or stable resonators for clock generation [1]. More recently, thanks to the sensitivity of the resonance frequency and the signal transmission delay to the physical environmental parameters, the use of SAW devices has been expanded to measure gas concentrations, pressures, forces, temperatures, strains or radiations. In this regard, SAW sensors have a great added value, being passive (batteryless) and potentially remotely readable (wireless) [2, 3]. By combining the latter properties with a small size device, a low-cost radio system and small antennas (provided that it is a high frequency device), new and exciting perspectives for wireless measurement processes and ID-Tag applications have emerged. Moreover, by choosing the proper materials, those sensors can be used without embedded electronics in hostile environments, for example under high temperature, under ionizing radiations or under high pressure (up to several thousand bar), where conventional wireless sensors cannot survive. However, one current bottleneck of the SAW sensor technology in these harsh

Experiments were carried out at MiNaLor clean-room platform which is partially supported by FEDER and Grand Est Region through the RaNGE project and at IJL TUBE-Davm equipment which is funded by the French PIA project "Lorraine Université d'Excellence" (ANR-15-IDEX-04-LUE). This work was supported by the Direction Générale de l'Armement (DGA), the ANR project "SAWGOOD" (ANR-18-CE42-0004-01), the CAPMAT project ("FEDER-FSE Lorraine et Massif Vosges 2014-2020" and ICEEL), the Ministry of Science and Higher Education of the Russian Federation (075-022020-1588), and the National University of Science and Technology MISIS (K2-2020-007). environments is the lack of a reliable packaging solution [4]. This technological barrier could be elegantly broken by the development of so-called "packageless" sensors. It has already been shown theoretically and experimentally that a packageless solution is achievable using Waveguiding Layer Acoustic Waves (WLAW) [5-8]. In a WLAW structure, the wave propagates in a low velocity layer "sandwiched" between two high acoustic velocity layers [5]. A WLAW packageless temperature sensor was achieved on a $\mathrm{Y}+128^{\circ}$ lithium niobate based AlN/ZnO/LN-Y128 trilayer [9] with a Temperature Coefficient of Frequency (TCF) as high as $-106 \mathrm{ppm} /{ }^{\circ} \mathrm{C}$.

In a previous study, an AlN/LN-Y128 bilayer, coupled with heavy Pt electrodes [10], was proposed as an alternative packageless high temperature sensor and theoretically analyzed using SDA-FEM-SDA [11]. This bilayer structure is advantageous over the AlN/ZnO/LNY128 trilayer [9] as only one additional layer has to be deposited over the LN-Y128 substrate and optimized. Moreover, $\mathrm{ZnO}$ has poor high temperature properties, whereas all the materials in AlN/IDT(Pt)/LNY128 can withstand high temperature operation at least up to $600^{\circ} \mathrm{C}[12-14]$.

In this paper, we aim at providing the experimental validation of the AlN/IDT(Pt)/LN-Y128 structure at frequencies over $2 \mathrm{GHz}$. Theoretical findings from [10] are first shortly described. Then the microfabrication, the AlN growth and the morphological and structural analyses are discussed. Electrical characterizations are compared with numerical analyses. Finally, the use as a packageless sensor and measurements up to $300^{\circ} \mathrm{C}$ are provided.

\section{RESULTS AND DISCUSSION}

\section{A. Peculiarities of the AlN/IDT(Pt)/LN-Y128 structure}

As a brief summary of [10], thanks to the use of thick and dense electrodes, Rayleigh-type acoustic waves can propagate along the AlN/LN-Y128 interface with a high velocity up to $4500 \mathrm{~m} / \mathrm{s}$, a high electromechanical coupling of 5.6\% and negligible leakage into the LN-Y128 substrate. With optimized Pt electrodes and AlN thicknesses, the velocity of this mode can be engineered in the interval between $4029 \mathrm{~m} / \mathrm{s}$ and $4644 \mathrm{~m} / \mathrm{s}$, i.e. between the slow and fast shear cut-off bulk acoustic wave (respectively written $V_{\text {B1-LN }}$ and $V_{\text {B2-LN }}$ ). In this interval, the Rayleigh-type wave does not couple with the slow shear bulk acoustic wave and does not leak in the substrate, due to

Cécile Floer, Omar Elmazria, Florian Bartoli, Jaafar Ghanbaja, and Sami Hage-Ali are with Université de Lorraine, CNRS, IJL, F-54000 Nancy, France (e-mail : cecile.floer@univ-lorraine.fr, sami.hage-ali@univ-lorraine.fr )

Natalya Naumenko is with National University of Science and Technology "MISIS", Moscow, Russia (e-mail : naumenko.natalya@gmail.com )

Thierry Aubert is with Université de Lorraine, CentraleSupélec, LMOPS, F57000 Metz, France (e-mail : thierry.aubert@univ-lorraine.fr ) 
anomalous properties of the LN-Y128. Higher velocities $\left(>\mathrm{V}_{\mathrm{B} 2-\mathrm{LN}}=\right.$ $4644 \mathrm{~m} / \mathrm{s}$ ) would result in strong leakage of the Rayleigh wave into the substrate, a phenomenon that is prevented in this structure by the use of optimized thick and dense electrodes that slow the wave. Assuming that a sufficient AlN thickness is used to protect the wave from environmental influence for packageless operation, i.e. about one wavelength $\lambda$, the wave velocity is expected to be suitable when the $\mathrm{Pt}$ thickness is close to $0.1 \lambda$.

\section{B. Microfabrication and structural analysis}

In order to validate this structure experimentally, a single port resonator structure with a wavelength $\lambda=1.7 \mu \mathrm{m}$ was patterned on a LN-Y128 substrate by electron beam lithography and ion beam etching (IBE) methods on a $\sim 170 \mathrm{~nm}(0.1 \lambda)$ sputtered Pt layer.

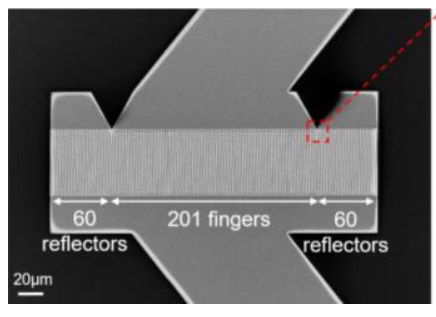

(a)

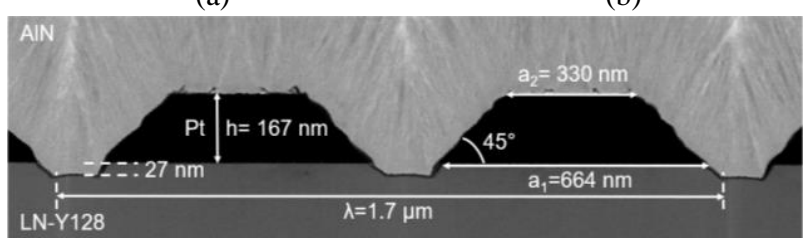

(c)

Fig. 1. (a) SEM micrograph and design of the platinum resonator. (b) Zoom on IDT fingers and the reflectors. (c) Cross-sectional STEM - Bright Field (BF) micrograph of the electrode geometry.

A top view of the platinum resonator and design information are shown in Fig. 1a. The bright color of the fingers' edge in the zoom image (Fig. 1b) indicates that the sides etching profile is not vertical. Cross sectional STEM images (Fig. 1c) show that the electrodes are of trapezoidal form, with titled sides with an angle of $45^{\circ}$, obtained repeatably due to masking effects during the IBE. The metallization ratio at the bottom $2 * a_{1} / \lambda$ is $78.1 \%$ and the bulk metallization ratio $\left(a_{1}+a_{2}\right) / \lambda$ - the important metric since it determines the mass loading is $58.4 \%$.

Subsequently, (002) oriented AlN was sputtered on the resonator. Two types of deposition were investigated: one (deposition $n^{\circ} 1$ ) without intentional substrate heating in a commercial machine and the other (deposition $\mathrm{n}^{\circ}$ ) at $480^{\circ} \mathrm{C}$ in a custom-built machine. The deposition parameters are summarized in Table I.

TABLE I: ALN DEPOSITION PARAMETERS

\begin{tabular}{lcc}
\hline \hline & Deposition 1 $(2.5 \mu \mathrm{m})$ & Deposition 2 $(3.2 \mu \mathrm{m})$ \\
\hline Sputtering & Alliance Concept DP650 & Custom-built \\
Target & $\mathrm{Al}(\varnothing$-inch $)$ & $\mathrm{Al}(\varnothing 2$-inch $)$ \\
Gases & $16 \mathrm{sccm} \mathrm{N}_{2}$ & $23.5 \mathrm{sccm} \mathrm{N} \mathrm{N}_{2}$ \\
Total pressure & $8 \times 10^{-3} \mathrm{mbar}$ & $6.6 \times 10^{-3} \mathrm{mbar}$ \\
Temperature & No intentional heating & $480^{\circ} \mathrm{C}$ \\
Target power & $200 \mathrm{~W}$ & $200 \mathrm{~W}$ \\
\hline
\end{tabular}

Then, morphological and nanostructural characterizations were carried out with scanning and transmission electron microscope (SEM and TEM, respectively). STEM micrographs in Fig. 2 show that the AlN film growth is affected by the trapezoidal shape of the electrodes for both depositions. For the low temperature deposition (Fig. 2a,2b), the grains arise from the tilted electrodes' sides to join further on. For the second deposition conducted at $480^{\circ} \mathrm{C}$, much less inhomogeneities can be observed (Fig. 2c,2d). The columns join each other much faster. Thus, the lower density areas do not appear over the entire thickness of the AlN film, highlighting a better crystallization. Moreover, an XRay diffraction rocking-curve analysis on the (002) orientation revealed a FWHM of $2.45^{\circ}$ leading to the conclusion that a good AlN quality can be achieved even on trapezoidal electrodes. (a)

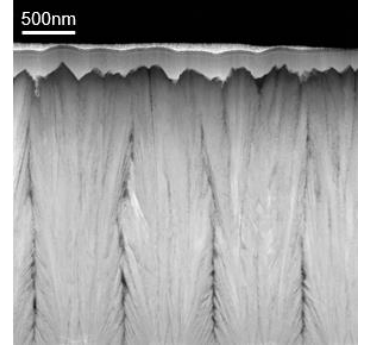

(c)

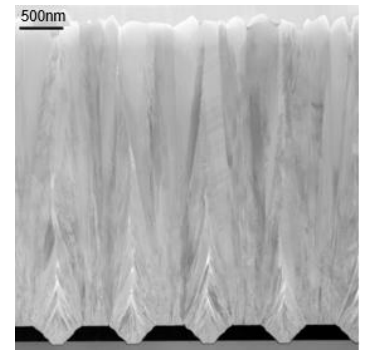

(b)

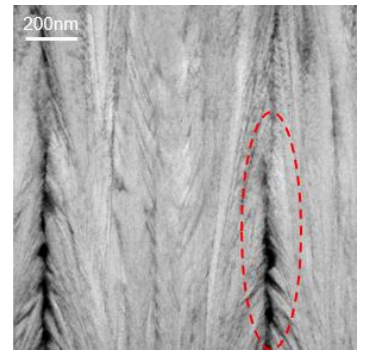

(d)

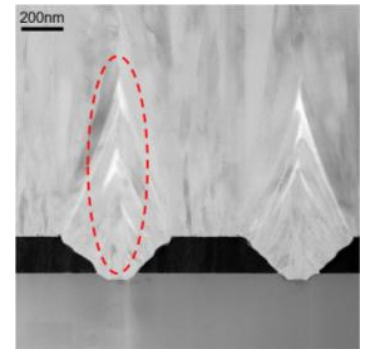

Fig. 2. Cross-sectional STEM - High Angle Annular Dark Field (HAADF) micrographs of the AlN film grown: (a),(b) without intentional heating and (c),(d) with a $480^{\circ} \mathrm{C}$ intentional heating.

\section{Electrical characterizations \& discussion}

The devices were electrically characterized before and after the AlN deposition using a network analyzer (VNA Agilent-N5230A) and the acoustic waves velocities were extracted from the admittance measurements. Simulations were performed taking into account the trapezoidal geometry of the electrodes using the SDA-FEM-SDA software [11], with constants from [15] for LN-Y128, and $\mathrm{c}_{11}=348 \mathrm{GPa}, \mathrm{c}_{12}=242 \mathrm{GPa}$ and a density of $21500 \mathrm{~kg} / \mathrm{m}^{3}$ for Pt.

Fig. 3 shows the velocities of the Rayleigh and Shear Horizontal (SH) modes in LN-Y128 with platinum electrodes, first without the AlN film (Fig. 3a), then with a thin AlN film in-between the Pt electrodes (Fig. 3b) and on top of them (Fig. 3c). In both cases with AlN (Fig. 3b and 3c) the Pt thickness is fixed at $0.1 \lambda$.

In the first simulated case (Fig. 3a), the mass loading effect is highlighted by the SAW velocities decrease due to the increase of the Pt thickness. Moreover, the antiresonance velocities of Rayleigh and $\mathrm{SH}$ waves, determined experimentally from the resonators show a good agreement with the simulations (see the square symbols on Fig. 3a). They have been identified respectively at $2692 \mathrm{~m} / \mathrm{s}$ and $2149 \mathrm{~m} / \mathrm{s}$ when the Pt electrodes thickness is equal to $0.1 \lambda$.

Then, the AlN elastic constants were fitted in order to match the experimental results from the $480^{\circ} \mathrm{C}$ AlN deposition (triangles Fig. 3c). The best agreement between simulations and experiments was obtained with the elastic stiffness constants $c_{11}=300 \mathrm{GPa}$ and $\mathrm{c}_{44}=86.5 \mathrm{GPa}$ and a density of $3250 \mathrm{~kg} / \mathrm{m}^{3}$.

These values where subsequently used to compute the velocities with increasing halN $/ \lambda$ (Fig. $3 b$ and $3 c$ ). The fast AlN layer explains the 
continuous increase of the velocities. The wave velocities hardly change as soon as the $h_{\text {AIN }} / \lambda$ relative thickness exceeds 1.0 , meaning that the influence of surface perturbations is very small.

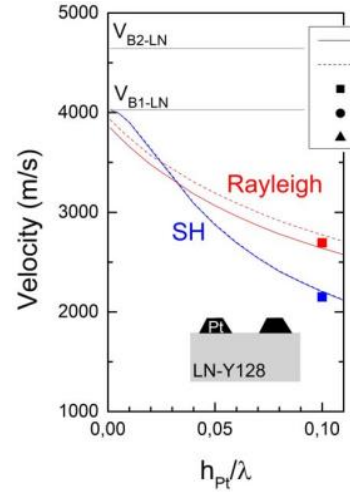

(a)

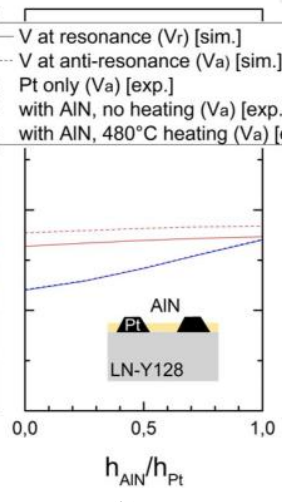

(b)

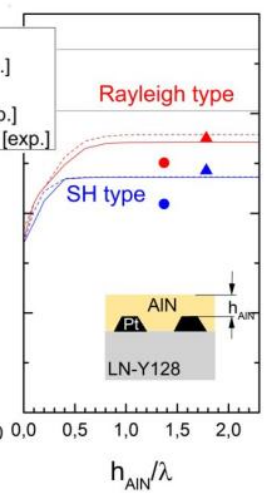

(c)
Fig. 3. Simulated and experimental velocities of two propagating modes in the AlN/IDT(Pt)/LN-Y128 bilayer structure as a function: (a) of the Pt thickness, (b) of the AlN thickness placed in-between or (c) on top of the electrodes. For (b) and (c) the Pt thickness is fixed at $0.1 \lambda$.

In Fig. $3 c$, even if the $h_{\text {AlN }} / \lambda$ ratio is a bit different, we can observe significant experimental velocities difference between the sample with AlN deposited at low temperature (circles) and at $480^{\circ} \mathrm{C}$ (triangles). This can be attributed to the morphological and crystalline differences visible in Fig. 2. This also means that AlN deposition parameters can be used to engineer the fine tuning of velocities at constant $\mathrm{Pt}$ thicknesses.

It is also noteworthy that, even with the $480^{\circ} \mathrm{C}$ deposition, experimental velocities are quite far from theoretical predictions from [10] (that used $\mathrm{c}_{11}=393 \mathrm{GPa}$ and $\mathrm{c}_{44}=130 \mathrm{GPa}$ for AlN), at similar mass loading (i.e. $3751 \mathrm{~m} / \mathrm{s}$ vs $4200 \mathrm{~m} / \mathrm{s}$, respectively, for the antiresonance of the Rayleigh type mode). From a design standpoint, this finding is actually highly beneficial, since thinner Pt electrodes can be used in order to reach velocities in the $4000-4500 \mathrm{~m} / \mathrm{s}$ interval. Indeed, a thinner Pt electrode would lead to a lower velocity decline and thus to higher final velocities and frequencies, after adding the AlN fast layer. Fabrication would also be easier and viscoelastic losses diminished. Assuming no significant change of the AlN film quality when it is deposited on thinner electrodes, the optimal Pt thickness is estimated to be about $0.05 \lambda$, with the same constants.

The wave confinement in the $\operatorname{AlN}\left(480^{\circ} \mathrm{C}\right) / \mathrm{IDT}(\mathrm{Pt}) / \mathrm{LN}-\mathrm{Y} 128$ bilayer structure has been tested by disturbing the surface of the structure with a very flexible elastomeric perturber named Solaris placed on top of it, like in [9]. The comparison of the perturbed and unperturbed $S_{11}$ frequency response is shown in Fig. 4. No influence of the elastomeric pertuber on the $S_{11}$ coefficient is observed, proving that the wave is confined inside the bilayer structure and consequently that the structure is suitable to achieve packageless devices.

To investigate the potential of the structure as a high temperature sensor, the resonance frequency of the AlN/IDT(Pt)/LN resonator was measured when increasing the temperature from 30 to $300^{\circ} \mathrm{C}$ (Fig. 5a). The base level and impedance matching difference between Fig. 4 and Fig. 5 a (case at $30^{\circ} \mathrm{C}$ ) results from the probe position variability on our ball connection which is needed to protect the pad access during the AlN deposition. The repetition of measurements and the probes' thermal expansion also caused the connection balls to fail after a few iterations. It was thus not possible to test the device at higher temperatures. However, a high TCF value $-138 \mathrm{ppm} /{ }^{\circ} \mathrm{C}$ is obtained for the Rayleigh type wave (Fig. 5b). Therefore, this bilayer structure is a good candidate for a packageless temperature sensor at high temperatures.

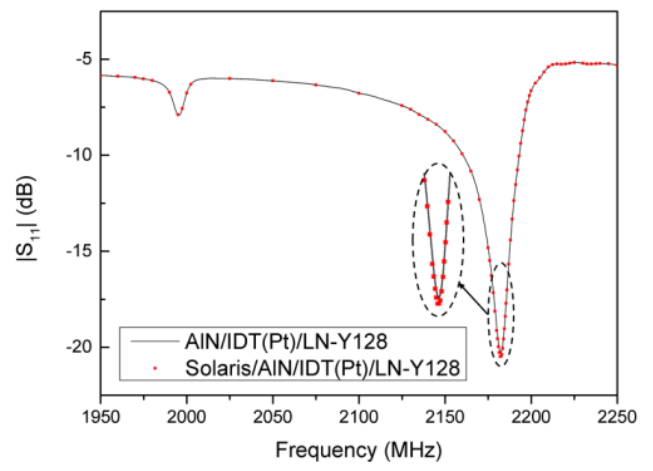

Fig. 4. $\mathrm{S}_{11}$ measurement of the $\operatorname{AlN}\left(480^{\circ} \mathrm{C}\right) / \mathrm{IDT}(\mathrm{Pt}) / \mathrm{LN}-\mathrm{Y} 128$ structure with and without a surface perturbation (Solaris elastomer) on top of the AlN layer.

(a)

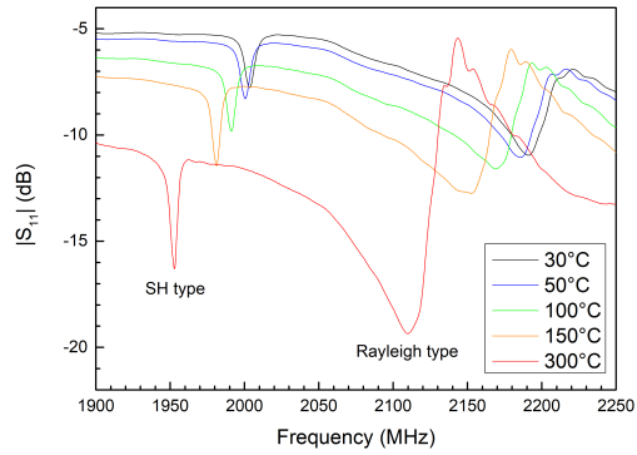

(b)

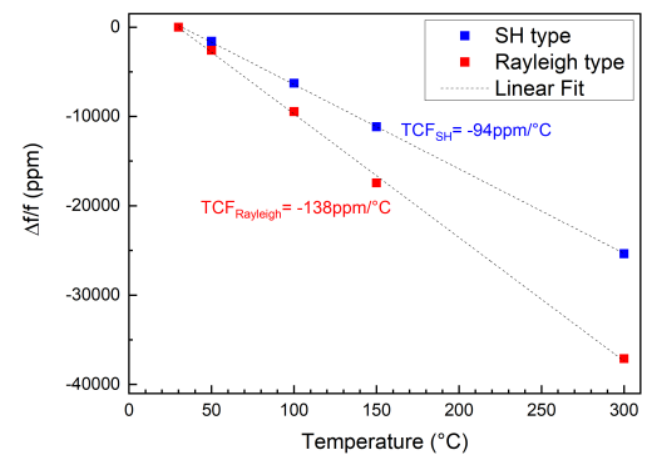

Fig. 5. (a) $\mathrm{S}_{11}$ measurements of the $\operatorname{AlN}\left(480^{\circ} \mathrm{C}\right) / \mathrm{IDT}(\mathrm{Pt}) / \mathrm{LN}-\mathrm{Y} 128$ structure between $30^{\circ} \mathrm{C}$ and $300^{\circ} \mathrm{C}$. (b) Relative frequency shifts with respect to the temperature of the $\mathrm{AlN}\left(480^{\circ} \mathrm{C}\right) / \mathrm{IDT}(\mathrm{Pt}) / \mathrm{LN}-\mathrm{Y} 128$ structure.

\section{CONCLUSION}

To summarize, the potential of the AIN/IDT(Pt)/LN-Y128 bilayer structure as a packageless sensor has been validated experimentally. The use of thick electrodes made from a dense metal such as platinum, placed on a LN-Y128 substrate and buried in a high velocity layer like AlN leads to a confined acoustic mode. A high sensitivity to the temperature was also observed making this bilayer structure promising for packageless high temperature sensing applications.

Future investigations will focus on the optimization of the $\mathrm{Pt}$ thickness and the AIN deposition parameters in order to reach optimal speeds, frequencies and loss mitigation. Other material choices are worth investigating [16]. This study could also be extended to reflective delay-lines (RDL). By combining a sensing and an identification functionality in a unique device, RDL become particularly interesting for the industry 4.0. 


\section{REFERENCES}

[1] C. Campbell, Surface acoustic wave devices for mobile and wireless communications. San Diego: Academic Press, 1998.

[2] W. Buff, S. Klett, M. Rusko, J. Ehrenpfordt, and M. Goroli, "Passive remote sensing for temperature and pressure using SAW resonator devices," IEEE Transactions on Ultrasonics, Ferroelectrics, and Frequency Control, vol. 45, no. 5, pp. 1388-1392, Sep. 1998, doi: $10.1109 / 58.726466$.

[3] C. Fu, O. Elmazria, F. Sarry, T. Mahalingam, S. S. Yang, and K. Lee, "Development of wireless, batteryfree gyroscope based on one-port SAW delay line and double resonant antenna," Sensors and Actuators A: Physical, vol. 220, pp. 270-280, Dec. 2014, doi: 10.1016/j.sna.2014.10.006.

[4] B. François, J.-M. Friedt, G. Martin, and S. Ballandras, "High temperature packaging for surface acoustic wave transducers acting as passive wireless sensors," Sensors and Actuators A: Physical, vol. 224, pp. 6-13, Apr. 2015, doi: 10.1016/j.sna.2014.12.034.

[5] K. Bhattacharjee, A. Shvetsov, and S. Zhgoon, "Packageless SAW Devices with Isolated Layer Acoustic Waves (ILAW) and Waveguiding Layer Acoustic Waves (WLAW)," in 2007 IEEE International Frequency Control Symposium Joint with the 21 st European Frequency and Time Forum, May 2007, pp. 135-140, doi: 10.1109/FREQ.2007.4319049.

[6] O. Elmazria, S. Zhgoon, L. Le Brizoual, F. Sarry, D. Tsimbal, and M. A. Djouadi, "AlN/ZnO/diamond structure combining isolated and surface acoustic waves," Appl. Phys. Lett., vol. 95, no. 23, p. 233503, Dec. 2009, doi: $10.1063 / 1.3270536$.

[7] O. Legrani, O. Elmazria, S. Zhgoon, P. Pigeat, and A. Bartasyte, "Packageless AlN/ZnO/Si Structure for SAW Devices Applications," IEEE Sensors Journal, vol. 13, no. 2, pp. 487-491, Feb. 2013, doi: 10.1109/JSEN.2012.2219692.

[8] F. Bartoli et al., "AlN/GaN/Sapphire heterostructure for hightemperature packageless acoustic wave devices," Sens. Actuators, A, vol. 283, pp. 9-16, Nov. 2018, doi: 10.1016/j.sna.2018.08.011.

[9] C. Floer et al., "AIN/ZnO/LiNbO 3 Packageless Structure as a Low-Profile Sensor for Potential On-Body Applications," IEEE Trans. Ultrason., Ferroelectr., Freq. Control, vol. 65, no. 10, pp. 1925-1932, Oct. 2018, doi: 10.1109/TUFFC.2018.2839262.

[10] N. Naumenko and P. Nicolay, "AlN/Pt/LN structure for SAW sensors capable of operating at high temperature," Appl. Phys. Lett., vol. 111, no. 7, p. 073507, Aug. 2017, doi: 10.1063/1.4985582.

[11] N. F. Naumenko, "Advanced numerical technique for analysis of surface and bulk acoustic waves in resonators using periodic metal gratings," Journal of Applied Physics, vol. 116, no. 10, p. 104503, Sep. 2014, doi: 10.1063/1.4895140.

[12] C. Floer et al., "SAW RFID Devices Using Connected IDTs as an Alternative to Conventional Reflectors for Harsh Environments," IEEE Trans. Ultrason., Ferroelectr., Freq. Control, vol. 67, no. 6, pp. 12671274, Jun. 2020, doi: 10.1109/TUFFC.2019.2943310.

[13] J. Streque et al., "Stoichiometric Lithium Niobate Crystals: Towards Identifiable Wireless Surface Acoustic Wave Sensors Operable up to $600^{\circ} \mathrm{C}$," IEEE Sensors Letters, vol. 3, no. 4, pp. 1-4, Apr. 2019, doi: 10.1109/LSENS.2019.2908691.

[14] T. Aubert et al. "Investigations on AlN/sapphire piezoelectric bilayer structure for high-temperature SAW applications," IEEE Trans. Ultrason., Ferroelectr., Freq. Control, vol. 59, no. 5, pp. 999-1005, May 2012, doi: 10.1109/TUFFC.2012.2285.

[15] J. Kushibiki et al. "Accurate measurements of the acoustical physical constants of $\mathrm{LiNbO}_{3}$ and $\mathrm{LiTaO}_{3}$ single crystals," IEEE Trans. Ultrason., Ferroelectr., Freq. Control, vol. 46, no. 5, pp. 1315-1323, Sept. 1999, doi: 10.1109/58.796136.

[16] P. Nicolay, N. Naumenko, T. Aubert, and H. Chambon, "Towards an AlScN-based packageless acoustic wave sensor with RFID capabilities, for applications above $350^{\circ} \mathrm{C}$," IEEE Ultrasonics Symposium 2018, doi:10.13140/RG.2.2.14675.02083 